OPEN ACCESS

Edited by:

Christophe Faure,

CHU Sainte-Justine Research Center,

Université de Montréal, Canada

Reviewed by:

Moftah Hussin Alhagamhmad, Al-Arab Medical University, Libya

Corentin Babakissa

Université de Sherbrooke, Canada

Allan Goldstein,

Massachusetts General Hospital, Harvard Medical School,

United States

*Correspondence:

Yu Shi

shiyu_chcmu@yahoo.com Jie Chen

jchen010@foxmail.com

Weihong Song

weihong@mail.ubc.ca

Tingyu $\mathrm{Li}$

tyli@vip.sina.com

Specialty section

This article was submitted to Pediatric Gastroenterology,

Hepatology and Nutrition,

a section of the journal

Frontiers in Pediatrics

Received: 23 May 2018 Accepted: 16 November 2018 Published: 03 December 2018

Citation:

Li C, Hu R, Hou N, Wang Y, Wang Z, Yang T, Gu Y, He M, Shi Y, Chen J, Song $W$ and $L i T$ (2018) Alteration of the Retinoid Acid-CBP Signaling Pathway in Neural Crest Induction Contributes to Enteric Nervous System Disorder.

Front. Pediatr. 6:382. doi: 10.3389/fped.2018.00382

\section{Alteration of the Retinoid Acid-CBP Signaling Pathway in Neural Crest Induction Contributes to Enteric Nervous System Disorder}

\author{
Cheng $\mathrm{Li}^{1,2}$, Rong $\mathrm{Hu}^{2}$, Nali Hou ${ }^{1}$, Yi Wang ${ }^{3}$, Zhili Wang ${ }^{3}$, Ting Yang ${ }^{1}$, Yan $\mathrm{Gu}^{1}$, Mulan $\mathrm{He}^{1}$, \\ Yu Shi ${ }^{2,4 *}$, Jie Chen ${ }^{1 *}$, Weihong Song ${ }^{2,5 *}$ and Tingyu $\mathrm{Li}^{1,2 *}$ \\ ${ }^{1}$ Children's Nutrition Research Center, Children's Hospital of Chongqing Medical University, Chongqing, China, ${ }^{2}$ Ministry of \\ Education Key Laboratory of Child Development and Disorders and Chongqing Key Laboratory of Translational Medical \\ Research in Cognitive Development and Learning and Memory Disorders, Children's Hospital of Chongqing Medical \\ University, Chongqing, China, ${ }^{3}$ Department of Gastrointestinal Surgery and Neonatal Surgery, Children's Hospital of \\ Chongqing Medical University, Chongqing, China, ${ }^{4}$ Clinical Laboratory, Children's Hospital of Chongqing Medical University, \\ Chongqing, China, ${ }^{5}$ Townsend Family Laboratories, Department of Psychiatry, University of British Columbia, Vancouver, BC, \\ Canada
}

Hirschsprung Disease (HSCR) and/or hypoganglionosis are common pediatric disorders that arise from developmental deficiencies of enteric neural crest cells (ENCCs). Retinoid acid (RA) signaling has been shown to affect neural crest (NC) development. However, the mechanisms underlying RA deficiency-induced HSCR or hypoganglionosis are not well-defined. In this report, we found that in HSCR patient bowels, the RA nuclear receptor RAR $\alpha$ and its interacting coregulator CREB-binding protein (CBP) were expressed in enteric neural plexuses in the normal ganglionic segment. However, the expression of these two genes was significantly inhibited in the pathological aganglionic segment. In a Xenopus laevis animal model, endogenous RAR $\alpha$ interacted with CBP and was expressed in NC territory. Morpholino-mediated knockdown of RAR $\alpha$ blocked expression of the NC marker genes Sox10 and FoxD3 and inhibited NC induction. The morphant embryos exhibited reduced nervous cells in the gastrointestinal anlage, a typical enteric nervous deficiency-associated phenotype. Injection of CBP mRNA rescued NC induction and reduced enteric nervous deficiency-associated phenotypes. Our work demonstrates that RAR $\alpha$ regulates Sox10 expression via CBP during NC induction, and alteration of the RA-CBP signaling pathway may contribute to the development of enteric nervous system disorders.

Keywords: retinoid acid signal, Sox10, CBP, neural crest, Hirschsprung disease

\section{INTRODUCTION}

Hirschsprung disease (HSCR) and/or hypoganglionosis are common congenital disorders of the enteric nervous system (1). The predominant feature of HSCR is a lack of enteric neurons in the end of the bowel; whereas hypoganglionosis refers to reduced enteric neurons throughout the entire bowel (1). Both conditions are due to developmental abnormities of the neural crest (NC) (2). The 
absence of nerve bodies that regulate the activity of the colon makes the affected intestinal segment unable to relax and pass stool, resulting in an obstruction. The neural crest (NC) is a group of transient pluripotent cells induced by complex morphogen signals at the neural-epidermis border (3). Border region genes such as Pax3, Zic1, Msx1, and Sox10 synergistically activate downstream specifier genes and accomplish NC induction (3-5). After induction, which is usually marked by FoxD3 or Slug expression, NC cells migrate out from the dorsal neural tube to different destinations where they develop into various tissues and organs such as pigment cells, cranial facial cartilage, heart outflow tract, and the enteric nervous system $(5,6)$.

Xenopus laevis is a versatile model for studying $\mathrm{NC}$ development because of its large size (embryo diameter $1 \mathrm{~mm}$ ), numerous embryos in each oviposition and easy feeding (79). The developmental processes and molecular mechanisms of NC appear to be similar between species (10-12). In Xenopus laevis, NC induction initiates during late gastrulation (stage 12 according to the Nieuwkoop developmental schedule). After neural tube closure, NC cells leave the neural tube in a rostral-caudal wave (12). The NC cells in Xenopus laevis can either invade the gut during early phase (Stage 25-33 straight gut) or later phases (stage 40-41, at the onset of coiling) (13). Similar to other species, the enteric neuron precursor cells in Xenopus laevis are mainly derived from the vagal region, with minor contributions from the sacral level. These enteric neural crest cells (ENCCs) migrate into the primitive gut following the ventromedial pathway (between somites and the neural tube/notochord) $(13,14)$.

Retinoid acid (RA) influences various physical and pathological processes by activating the retinoic acid nuclear receptors (RARs/RXRs) (15). This heterodimer hormone receptor recruits histone acetyltransferase (HAT) or histone deacetylase (HDAC) and thereby activates or represses gene expression, respectively (16-19). CREB-binding protein (CBP) is a histone acetyltransferase (HAT) that associates with and acetylates transcriptional regulators and chromatin (20). CBP works as a coregulator of RAR hormone receptors (21-23).

The RA signaling pathway has long been known to regulate gastrointestinal nervous system development (24). In mouse models, targeted inactivation of RALDH2, a key enzyme responsible for RA synthesis, disrupts enteric nervous system development (25). Migrating NC cells express RAR $\alpha$ which binds the RA ligand secreted by the paraxial mesoderm (26). This interactions triggers Ret (27), a key component necessary for enteric NC survival, migration and colonization (28-33). Normal Ret expression requires Sox10 binding at its upstream promoter region (34). Mutations in Sox10 have been reported to affect HSCR development (34). Whether and when RA signaling regulates Sox10 in enteric nervous system pathogenesis are not yet known. In this study, we found that $\mathrm{RAR} \alpha$ regulates Sox10 expression via CBP during NC induction, and alteration of the RA-CBP signaling pathway may contribute to the development enteric nervous system disorders.

\section{MATERIALS AND METHODS}

\section{Tissue and Patients}

This study was carried out in accordance with the recommendations of the Ethics Committee of Children's Hospital of Chongqing Medical University. The protocol was approved by the Ethics Committee of Children's Hospital of Chongqing Medical University. All subjects gave written informed consent in accordance with the Declaration of Helsinki. Colon tissue from the normal and spastic segments of patients was obtained after operation on 7 boys and 2 girls that were between 2 months to 4 years old. They had no family history of the disease. Two of the cases were the short-segment type. Tissue samples were fixed with $4 \%$ paraformaldehyde and then imbedded in paraffin for immunofluorescence.

\section{Immunofluorescent Staining}

Colon samples were sectioned at a thickness of $4 \mu \mathrm{m}$ and pretreated with citrate buffer solution ( $\mathrm{pH}$ 6.0) for $30 \mathrm{~min}$ at $95^{\circ} \mathrm{C}$. After blocking in 5\% BSA for $30 \mathrm{~min}$, sections were incubated with primary antibodies (goat anti-RAR $\alpha$, Abcam ab28767, UK; rabbit anti-CBP, Novus NB100-91721, USA; mouse anti- $\beta$-Tubulin III, Santa Cruz sc-80005, USA; rabbit antiNeuN, Millipore MABN140, USA; mouse anti-NeuN, Millipore MAB377) overnight at $4^{\circ} \mathrm{C}$. Sections were washed with PBS and incubated in corresponding secondary antibodies (donkey anti-goat Alexa Fluor ${ }^{\circledR} 594$, Abcam ab150129; donkey antirabbit Alexa Fluor ${ }^{\circledR} 594$, Abcam ab150076; donkey anti-rabbit Alexa Fluor ${ }^{\circledR} 488$, Abcam ab150073; chicken anti-mouse Alexa Fluor ${ }^{\circledR} 488$, Invitrogen 1696214, USA; goat anti-rabbit Dylight ${ }^{\circledR} 488$ Abbkine A23220,USA; goat anti-mouse Dylight ${ }^{\circledR} 594$, Abbkine A23410) for $1.5 \mathrm{~h}$ at $22^{\circ} \mathrm{C}$.

\section{RNA Extraction and Real-Time PCR}

Total RNA samples were extracted from the colon of patients using the TriZol ${ }^{\mathrm{TM}}$ Total RNA Extraction kit (Ambion, USA). mRNA was reverse-transcribed into cDNA using the PrimeScript $^{\mathrm{TM}}$ RT Reagent kit (TaKaRa Bio, Japan), and realtime PCR reactions were carried out using a RealMasterMix ${ }^{\mathrm{TM}}$ kit (TaKaRa Bio). Primer sequences were: Human RAR $\alpha$ forward: $5^{\prime}$ tcc gcc gcagcatccagaagaacat, reverse: $5^{\prime}$-actcgg gct tgg gca cct cct tctt; Human CBP forward: $5^{\prime}$-acccaggcctcctcaatagt, reverse: $5^{\prime}$-tgg agtagggta cggcattc; and Human $\beta$-actin forward: $5^{\prime}$-gtgaaggtgaca gcagtcggtt, reverse: $5^{\prime}$-gagaagtggggtggcttttagga.

\section{Plasmid Construction}

Xenopus laevis RAR $\alpha$ and CBP coding regions were obtained by PCR and cloned into the pGMT vector to prepare probes. The following primers were used: Xenopus laevis RAR $\alpha$ forward $5^{\prime}$-cag cctattccegtgcca and, reverse $5^{\prime}$-catcgtgtcggtctgtcctt; and Xenopus laevis CBP forward 5 -gaatcc ttacctttcgtcagcc and reverse $5^{\prime}$-cagccggatggc aatggaag. Full-length Xenopus laevis $\mathrm{RAR} \alpha$ and CBP open reading frames were cloned into the pCS2+ expression vector for rescue experiments. The rescue plasmids do not contain the sequences targeted by RAR $\alpha$ - and CBPspecific morpholino oligomers (MOs). The following primers were used for full length rescue: RAR $\alpha$ : sense $5^{\prime}$-ccggaattccag cct 
attcccgtgcca, reverse $5^{\prime}$-tgctctagacat cgtgtccgtctgtcctt; and CBP: sense $5^{\prime}$-atttaggtgacactatag, reverse $5^{\prime}$-attaaccctcactaaaggga.

\section{Microinjection, Rescue, and Whole-Mount in situ Hybridization}

This study was performed in accordance with the recommendations of the Animal Experimentation Ethical Committee of Chongqing Medical University. in vitro fertilization and culture of embryos, whole-mount in situ hybridization, and microinjection were performed as described previously (35). All MOs were designed and purchased from GeneTools LLC (USA). The sequences of MOs were: RAR $\alpha-M O$ $5^{\prime}$-cgtccacat tctcatacatcctaaa; CBP-MO $5^{\prime}$ - gttctcggccatcttcactcc tttc; and Control-MO $5^{\prime}$ - cctcttacctcagttacaatttata. RAR $\alpha-\mathrm{MO}$ (6-24 ng per cell) or CBP-MO (10 ng per cell) were injected in two-cell or four-cell stage embryos unilaterally, and full length RAR $\alpha$ or CBP mRNA (100-300 pg) were used for rescue. LacZ was coinjected to trace the injected sides. Previously reported probes were used for in situ hybridization to detect the expression of Pax3, Zic1 (36), FoxD3 (37), Sox10 (38), and $\mathrm{N}$-tubulin (39).

\section{Coimmunoprecipitation and Western Blotting}

Embryos were lysed with 1\% Triton-X100 in phosphatebuffered saline containing a protease inhibitor cocktail. For immunoprecipitation analysis, $600-1200 \mu \mathrm{g}$ of protein was incubated with the anti-CBP antibody (Abcam ab50702, UK) or IgG and protein A/G plus agarose beads (Santa Cruz sc-2003, USA) overnight at $4^{\circ} \mathrm{C}$. The immunoprecipitation reactions were washed five times and boiled for western blotting. Approximately 80-120 $\mu \mathrm{g}$ of protein was resolved on 10\% SDS-PAGE gels and transferred to $0.45 \mu \mathrm{m}$ PVDF membranes. All membranes were blocked in 5\% marvel milk in TBST and then incubated with appropriate antibodies at $4^{\circ} \mathrm{C}$ overnight. Primary antibodies were used to detect RAR $\alpha$ (Novus NBP2-45516, USA) and CBP (Novus NB100-91721).

\section{Statistical Analyses}

All experiments were carried out at least three times. The Student's $t$-test was used, and $p<0.05$ was considered to be statistically significant.

\section{RESULTS}

\section{Absent RAR $\alpha$ and CBP Expression in Pathological Aganglionic Segments of HSCR Patients}

To explore roles of RAR $\alpha$ and CBP in enteric nervous system development, we first investigated the enteric expression patterns in excision bowels from 9 cases HSCR in children. Each bowel contains ganglionic (normal control) and aganglionic (pathological) segments. All 9 cases exhibited similar expression patterns. In the ganglionic segments, NC-derived ganglion cells expressed clear NeuN protein (Figure 1B red), consistent with previous reports $(40,41)$. Cell nuclear was stained by DAPI (Figures 1C,G,K,O,S,W). Beta-tubulin III and NeuN displayed partially overlapping expression in the merged panel (Figure 1D). CBP-positive cells (Figure 1F green) overlapped perfectly with NeuN-expressing cells (Figures 1E,H), suggesting that ganglion cells expressed CBP protein. These ganglion cells were arranged along the nerve fibers as detected by $\beta$-tubulin III staining (Figure 1A green) (42, 43). In addition, RAR $\alpha$ protein (Figure 1M) exhibited the same expression pattern as $\beta$-tubulin III (Figures 1N,P). In the aganglionic distal bowel, neither NeuN (Figure 1I, dash line) nor CBP (Figure 1J, dash line) was observed in the neural plexus (Figure 1L, dash line). CBP was weakly expressed in the muscularis propria region (Figure 1J). Previous work described decreased expression levels of $\beta$-tubulin in HSCR patients (44). In our study, expression of $\beta$-tubulin III (Figure 1R, dash line) as well as RAR $\alpha$ (Figure 1Q, dash line) was diminished in the aganglionic segments. Similar to the protein level, the ratio of mRNA levels in pathogenic aganglinoic segments to normal ganglionic segments are 0.459 \pm 0.273 for $\operatorname{RAR} \alpha\left({ }^{*} P<0.05, n=9\right)$ and $0.569 \pm 0.196$ for CBP $\left({ }^{*} p<0.05, n=9\right)$ (Figure 1Y). The partially overlapping expression pattern of RAR $\alpha$ and CBP in the normal ganglionic segment (Figures $\mathbf{1 U}-\mathbf{X}$ ) and their shared diminishment in the pathological aganglionic segment suggests that RAR $\alpha$ and CBP might play a role in enteric ganglion cell development and/or function.

\section{Expression of RAR $\alpha$ and CBP in the Developing NC Cells}

Enteric ganglion cells derive from NC cells. To explore the involvement of RAR $\alpha$ and CBP in NC development, we first checked the expression pattern of these two genes in Xenopus laevis. RAR $\alpha$ and CBP proteins shared similar temporal expression patterns during early embryonic development. Both RAR $\alpha$ and CBP proteins were maternally expressed (relative expression level at stage 2 designated as 1 , and the following numbers represent protein intensity ratios to stage 2), and the levels increased at the onset of the midblastula-transition (stage 7) (RAR $\alpha 1.46 \pm 0.39$, CBP 1.17 \pm 0.33 ) when zygotic transcription begins. Their expression peaked at the beginning of gastrulation (stage 10) (RAR $\alpha$ $2.57 \pm 1.08$, CBP $1.35 \pm 0.43)$ followed by a slight decrease at the later neurula stage (stage 22) (RAR $\alpha 1.92 \pm 1.16$, СBP $0.88 \pm 0.14 \mathrm{P}$ ) (Figure 2A). Whole mount in situ hybridization assays revealed the localization of RAR $\alpha$ and CBP in the animal hemisphere at stage 2 (Figures 2B,G) and stage 7 (Figures $2 \mathbf{C}, \mathbf{H}$ ). At the neural-plate stage, RAR $\alpha$ and CBP were expressed in the neural plate (Figures 2D,I, white asterisk) and plate-epidermis border region (Figures 2D,I, black arrowhead) where NC is induced. After neural tube closure, the branchial arch, which is composed of migrating NC cells, expressed RAR $\alpha$ (Figures 2E,F, red arrowhead) and CBP (Figures 2J,K, red arrowhead). Our data suggest that $\mathrm{RAR} \alpha$ and $\mathrm{CBP}$ are expressed in the developing $\mathrm{NC}$ cells. 

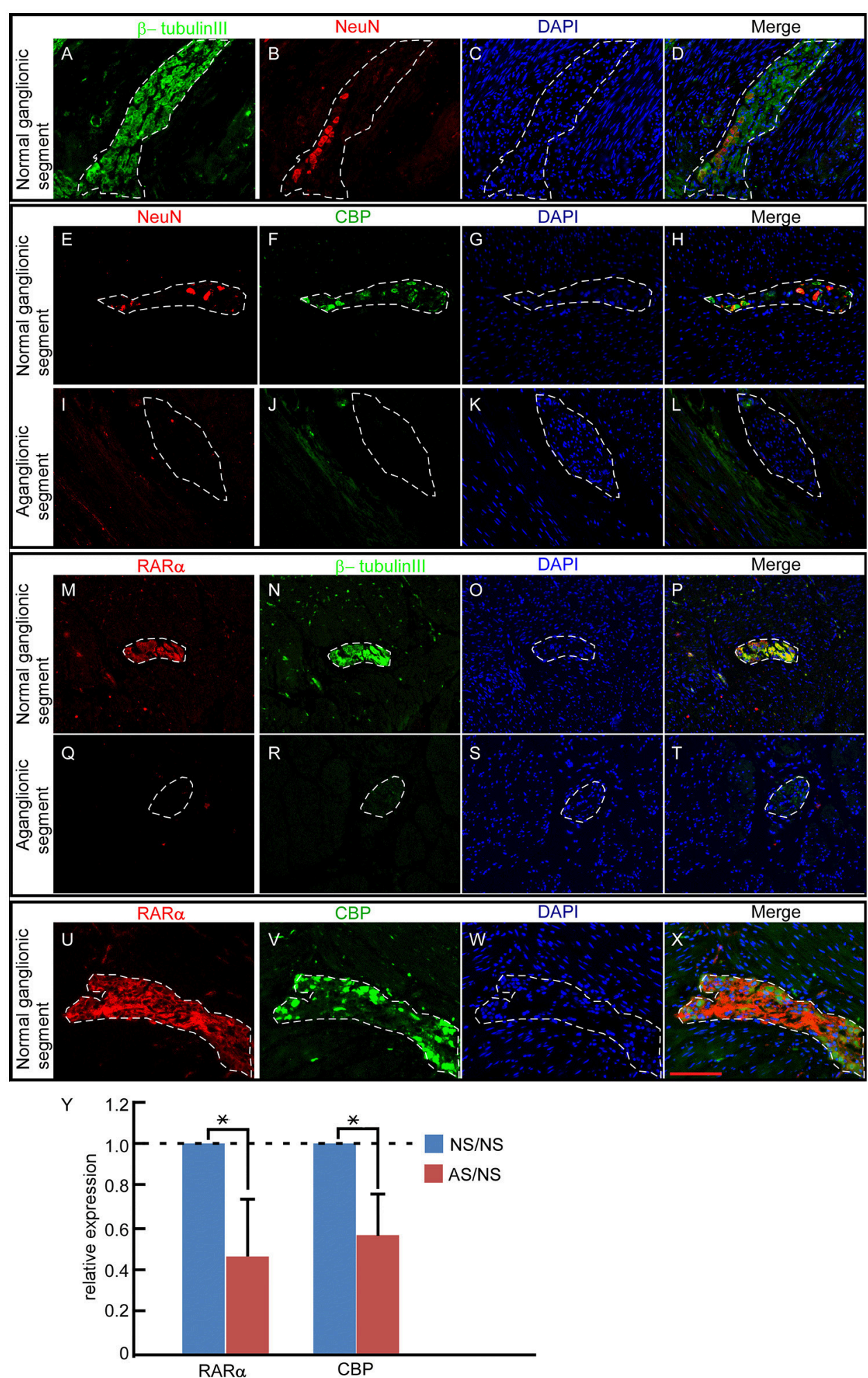

FIGURE 1 | RAR $\alpha$ and CBP expression patterns in the enteric nervous system of HSCR patients. (A-D) In normal ganglionic segments, $\beta$-tubulin III (green)-labeled nerve fibers (A) and NeuN protein (red) indicate ganglion cells (B). DAPI-stained cell nuclei (C) $\beta$-tubulin III and NeuN displayed partially overlapping expression patterns in the neural plexus, as shown in the merged panel (D). (E-H) In normal ganglionic segments, CBP protein (green) (F)-labeled ganglion cells are detected by 
FIGURE 1 I colocalization (H) with NeuN (red) (E). (I-L) In aganglionic segments, NeuN (I) and CBP (J) are absent within the neural plexus, and CBP is expressed faintly in the muscularis propria of the colon, as shown in the merged panel (L). (M-P) In normal ganglionic segments, RAR $\alpha$ (M) colocalizes with $\beta$-tubulin III (N), as shown in the merged panel (P). (Q-T) RAR $\alpha(\mathbf{Q})$ is absent and $\beta$-tubulin III (R) is weakly expressed in aganglionic segments. (U-X) In normal ganglionic segments, $\mathrm{RAR} \alpha \mathbf{( U )}$ labels nerve fibers and CBP (V) indicates ganglion cells. (X) Both proteins display partially overlapping patterns within the neural plexus. (Y) RAR $\alpha(0.459 \pm$ $0.273)$ and CBP $(0.569 \pm 0.196)$ transcripts were significantly lower in aganglionic segments than in normal regions. NS, normal ganglionic segments; AS, aganglionic segments. Numbers in brackets indicate the relative expression ratio of pathogenic aganglionic segments to normal ganglionic segments. Dashed lines encircle the neural plexus. ${ }^{\star \star} P<0.01$, with $T$-test. Scale bar: $100 \mu \mathrm{m}$.

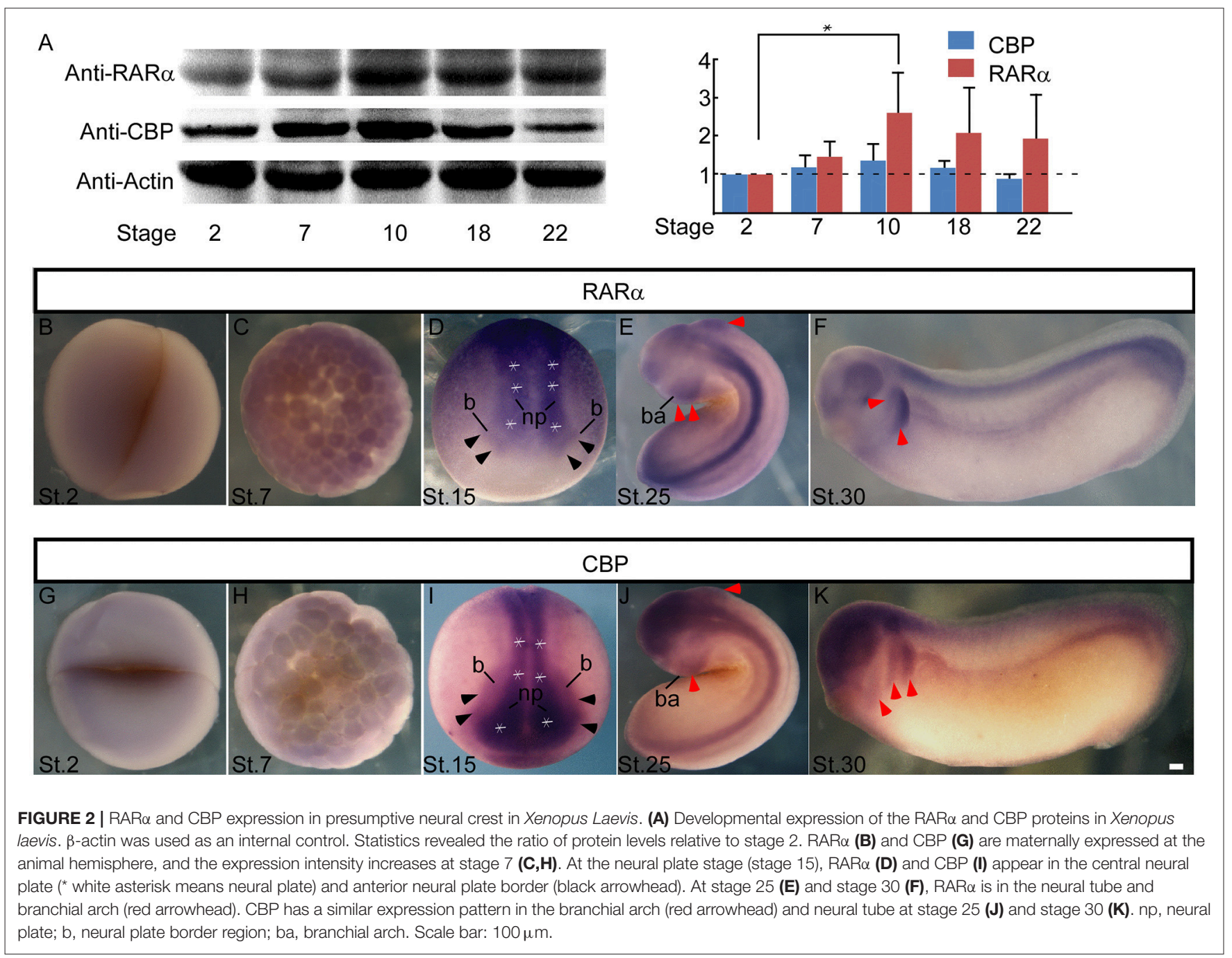

\section{RAR $\alpha$ and CBP Are Required for Enteric Nervous Cell Development}

Our data in HSCR patients indicated that RAR $\alpha$ and CBP proteins were absent in pathological aganglionic segments, suggesting that $R A R \alpha$ and CBP may participate in enteric nervous system development. To provide functional evidence, we knocked down RAR $\alpha$ and CBP translation with microinjection of specific morpholinos and examined enteric nervous system development in a Xenopus laevis model. There are two RAR $\alpha$ isoforms in Xenopus laevis, and RAR $\alpha 2$ is the only detectable isoform after gastrulation (45). Therefore, we designed a morpholino targeting $\mathrm{RAR} \alpha 2$ (designated as $\mathrm{RAR} \alpha$ in the following text). The RAR $\alpha 2$ - and CBP-specific morpholinos efficiently knocked down RAR $\alpha$ expression (Figures $\mathbf{3 A}, \mathbf{H}$ ) and inhibited CBP expression (Figure 3B) at stage 15, respectively. We evaluated enteric nervous system development by calculating the colony of enteric neural precursors/NC cells (labeled with Sox10) (46) and differentiated enteric nervous cells (labeled with $\mathrm{N}$-tubulin) (39) in the gut anlage (stage 41). In control embryos, the number of Sox10-labeled precursor cells was $17.3 \pm 6.66$ per gut (Figures 3G,K), while there were 93.6 $\pm 4.04 \mathrm{~N}$-tubulinpositive spots in each embryo gut anlage (Figures $3 \mathrm{C}, 3 \mathrm{~K}$ ). Both RAR $\alpha$ and CBP knock-down embryos displayed greatly reduced enteric neuron precursor cells (Sox10-positive blue spots $5.33 \pm 0.57$ /gut in CBP morphant and $5.66 \pm 2.08$ /gut in RAR $\alpha$ morphant) (Figures 3H,I,K). The colony numbers of 


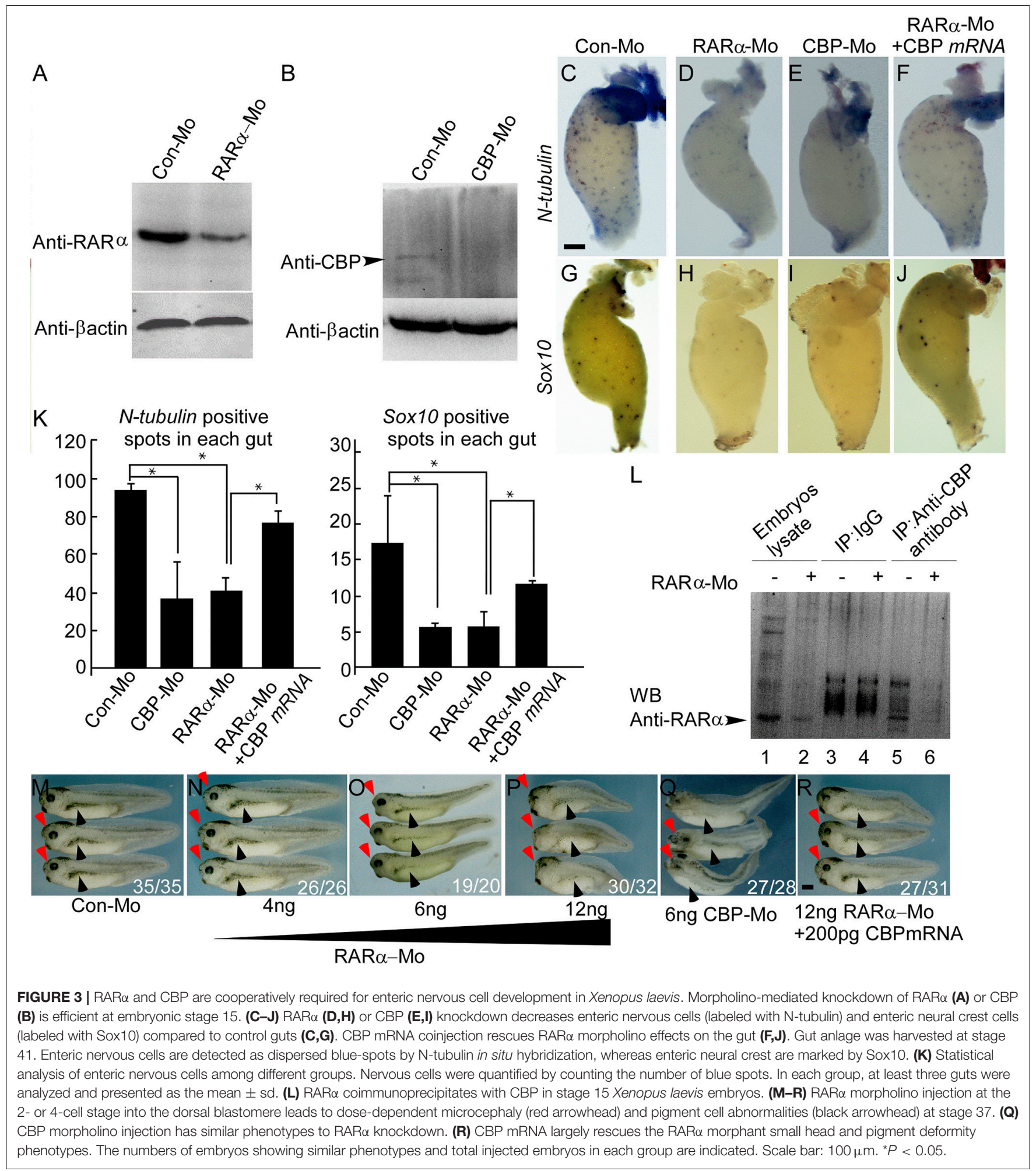

differentiated nervous cells, as illustrated by $\mathrm{N}$-tubulin in situ hybridization, were consequently decreased (positive blue spots $41.3 \pm 7.23$ /gut in RAR $\alpha$ morphants, Figures 3D,K and $37 \pm$ 19.2/gut in CBP morphants Figures 3E,3K) in the developing gut anlage $(P<0.05)$, which is a hypoganglionosis-like phenotype. Coinjection of CBP mRNA significantly rescued Sox10-(11.7 $\pm 0.58)$ and $\mathrm{N}$-tubulin-(77 \pm 6.24$)$ positive colony numbers in RAR $\alpha$ morphant embryos $(P<0.05)$ (Figures 3F,J). We also observed microcephaly (Figures $3 \mathbf{M}-\mathbf{Q}$ red arrowhead) and pigment cell abnormalities (Figures 3M-Q black arrowhead) in RAR $\alpha$ and CBP knock-down embryos. The severity of RAR $\alpha$ MO phenotypes was dose-dependent. 


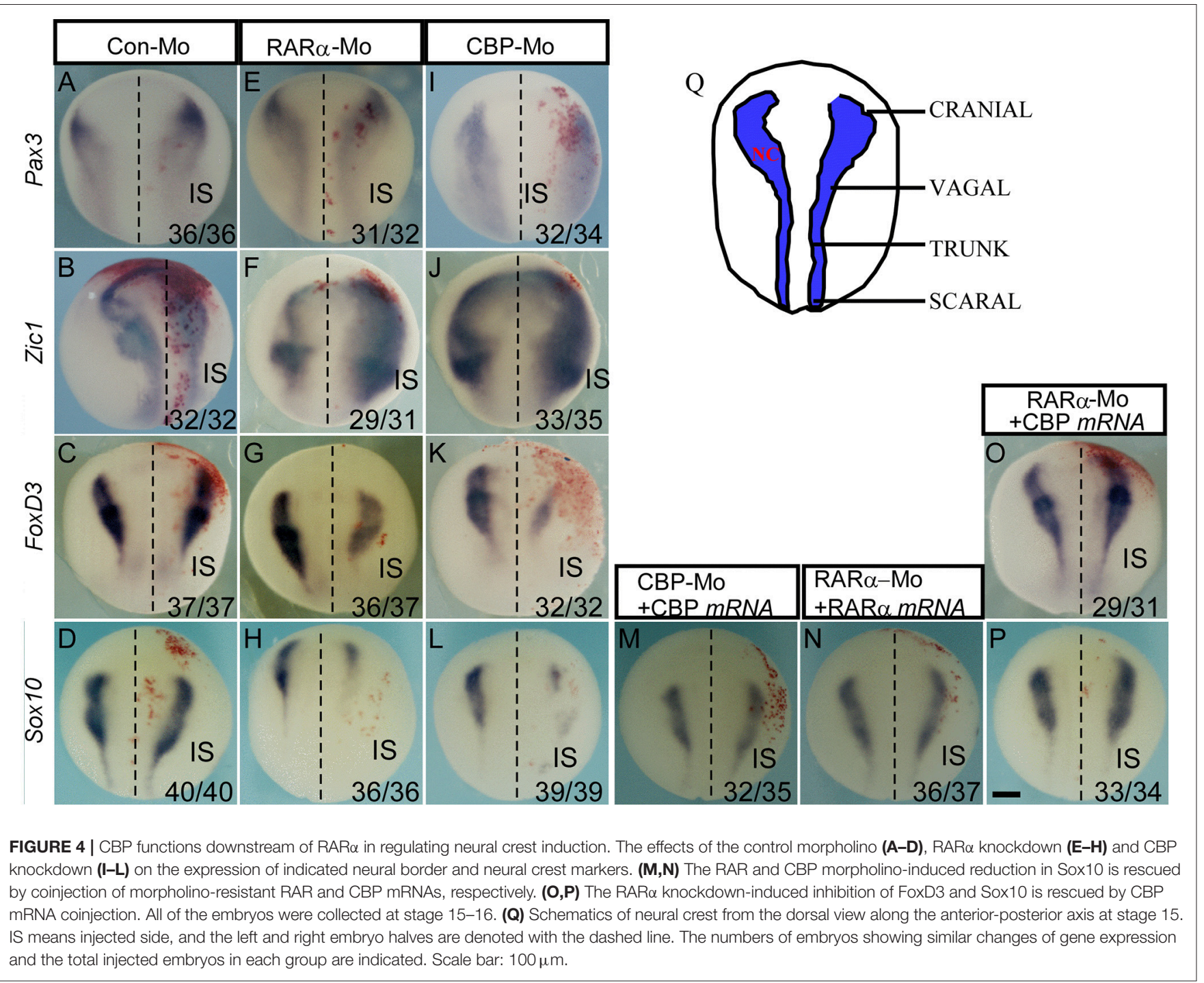

Previous work in our lab identified an interaction between RAR $\alpha$ and CBP in a rat model (47). It has long been known that the RA receptor regulates CBP expression (48). We found that Xenopus laevis CBP co-immunoprecipitated with $\mathrm{RAR} \alpha$ (Figure 3L). Microinjection of CBP mRNA rescued the RAR $\alpha$ morphant phenotypes in the head, pigment cells, and enteric nervous system (Figures 3R,F,J).

\section{CBP Functions Downstream of RAR $\alpha$ in Regulating NC Induction}

We demonstrated that developing NC cells express CBP and $\operatorname{RAR} \alpha$, and inhibition of CBP and $\operatorname{RAR} \alpha$ expression causes developmental abnormities of NC-derived enteric nervous cells, cranial facial structures, and pigment cells. We next investigated the temporal requirements and functions of RAR $\alpha$ and CBP in NC development. At stage 15 (the NC induction stage), knockdown of either CBP or RAR $\alpha$ did not affect Pax3 expression (Figures 4E,I) but did expand the Zic1-expression domain (Figures 4F,J). Sox10 (Figures 4H,L) and FoxD3 (Figure 4G,K) expression were inhibited in the morpholino-injected side. There was no effect of control morpholino on Pax3/ Zic1/ FoxD3/ Sox10 (Figures 4A-D). Such inhibition affects all NC regions including the cranial, vagal, trunk, and sacral areas as shown in the schematic (Figure 4Q) and subsequently affects enteric NC development. In addition, reduced expression of Sox10 and FoxD3 in RAR $\alpha$ and CBP morphants was rescued by exogenous injection of morpholino-resistant RAR $\alpha$ and CBP mRNAs (Figures $4 \mathbf{M}, \mathbf{N}$ ). NC induction is marked by FoxD3 and/or Slug expression $(37,49,50)$, which lead to the expression of numerous downstream transcription factors, such as Sox10 (51). Our data suggests that knockdown of either RAR $\alpha$ or CBP affects NC induction. CBP mRNA coinjection rescues the reduced Sox10 (Figure 4P) and FoxD3 (Figure 40) expression patterns in RAR $\alpha$ morphants. Such rescue indicates that CBP functions downstream of $\mathrm{RAR} \alpha$ during NC induction and partially accounts for rescue of the enteric nervous system development phenotypes at later stages. 


\section{DISCUSSION}

In this work, we first demonstrated that RAR $\alpha$ and CBP are normally expressed in the gastrointestinal ganglionic segment. In pathogenic aganglionic segments, RAR $\alpha$ and CBP are reduced or even absent, suggesting potential functions for these proteins in development and/or maintenance of the enteric nervous system. The gastrointestinal nervous system derives from NC (52). In chick, NC cells express RAR $\alpha$ (26). Our work in Xenopus laevis revealed that RAR $\alpha$ and its interactor CBP are expressed in the NC territory. The similar expression patterns of RAR $\alpha$ in different species suggests evolutionarily conserved roles for RA signaling in NC and enteric nervous system development.

Previous studies have showed that RA signaling affects cell polarization, lamellipodia formation and PTEN regulation, thereby affecting enteric NC migration (24). Inhibition of RA synthesis (25) or deletion of the vitamin A-binding protein (24) blocks NC migration and leads to HSCR-associated phenotypes. In this study, knockdown of either RAR $\alpha$ or its interactor CBP leads to failure of NC induction detected with reduced Sox10 and FoxD3 expression at the border region. Both morphant embryos also presented anterior-posterior body axis deformities and reduced anterior structures, consistent with previously reported RAR $\alpha$ morphant phenotypes (53). NC cells contribute to craniofacial cartilage genesis, which defines the scale of anterior head structures in the embryonic stage. Thus, abnormal NC induction caused by RAR $\alpha$ or CBP morpholinos accounts for the previously reported small head phenotype. At later stages (stage 41), both morphant embryos showed decreased enteric neural precursors/ NC cells and differentiated enteric nervous cells, which is a classic HSCR-associated phenotype. Injection of CBP mRNA rescued Sox10 and FoxD3 expression at the time of NC induction time and rescued subsequent enteric nervous system defects in RAR $\alpha$ morphants. These data clearly indicate that CBP functions downstream of RAR $\alpha$ in regulating Sox 10 expression during $\mathrm{NC}$ induction.

\section{REFERENCES}

1. Hirschsprung H. Stuhlträgheit neugeborener infolge von dilatation und hypertrophic des colons. Jb Inderheilk (1888) 27:1.

2. Butler Tjaden NE, Trainor PA. The developmental etiology and pathogenesis of Hirschsprung disease. Transl Res. (2013) 162:1-15. doi: 10.1016/j.trsl.2013.03.001

3. Milet C, Monsoro-Burq AH. Neural crest induction at the neural plate border in vertebrates. Dev Biol. (2012) 366:22-33. doi: 10.1016/j.ydbio.2012.01.013

4. Stuhlmiller TJ, Garcia-Castro MI. Current perspectives of the signaling pathways directing neural crest induction. Cell Mol Life Sci. (2012) 69:371537. doi: 10.1007/s00018-012-0991-8

5. Shyamala K, Yanduri S, Girish HC, Murgod S. Neural crest: the fourth germ layer. J Oral Maxillofac Pathol. (2015) 19:221-9. doi: 10.4103/0973-029X.164536

6. Bhatt S, Diaz R, Trainor PA. Signals and switches in Mammalian neural crest cell differentiation. Cold Spring Harb Perspect Biol. (2013) 5:a008326. doi: $10.1101 /$ cshperspect.a008326

7. Khedgikar V, Abbruzzese G, Mathavan K, Szydlo H, Cousin H, Alfandari D. Dual control of pcdh81/PCNS expression and function in Xenopus laevis neural crest cells by adam13/33 via the transcription factors tfap2alpha and arid3a. Elife (2017) 6:e26898. doi: 10.7554/eLife.26898

8. Vega-Lopez GA, Cerrizuela S, Aybar MJ. Trunk neural crest cells: formation, migration and beyond. Int J Dev Biol. (2017) 61:5-15. doi: $10.1387 / \mathrm{ijdb} .160408 \mathrm{gv}$
It has been shown that RA binds RAR $\alpha$ and then activates Ret (27). Activation of Ret gene expression requires Sox10 binding at the Ret promoter region (34). Ret is an indispensable gene in enteric NC development. Our data demonstrate the mechanism by which RAR $\alpha$ triggers Ret expression in HSCR pathogenesis. During NC induction, RAR $\alpha$ regulates Sox 10 expression via CBP, and subsequently Sox 10 binds the Ret upstream promoter region and initiates Ret transcription.

Based on the present data, RA signaling regulates enteric NC development at two stages: it first controls NC induction and later regulates enteric NC migration. Our study indicates that vitamin $\mathrm{A}$ and related metabolites may be risk factor for the penetrance and expressivity of HSCR disease. Optimizing maternal nutrition levels may prevent enteric neural system disorders.

\section{AUTHOR CONTRIBUTIONS}

CL, YS, TL, and WS conceived and designed the experiments. CL, YS, RH, NH, YW, ZW, TY, YG, MH, and JC performed the experiments. CL, YS, JC, TL, and WS analyzed and contributed reagents, materials, or analysis tools. CL, YS, JC, TL, and WS wrote the paper. All authors reviewed the manuscript.

\section{ACKNOWLEDGMENTS}

The authors thank Dr. Bingyu Mao for providing experimental reagents. This work was supported by a grant from the National Natural Science Foundation of China (81471518, to TL; 81771596 to YS), Chongqing Science and Technology Committee (CSTC2015jcyjBX0095 to YS), Chongqing Municipal Bureau of Health and Family Planning Project (2015MSXM036 to YS), and Canadian Institutes of Health Research (CIHR) Grant TAD-117948 (WS). WS holds the Tier 1 Canada Research Chair in Alzheimer's Disease.

9. Shellard A, Szabó A, Trepat S, Mayor R. Supracellular contraction at the rear of neural crest cell groups drives collective chemotaxis. Science (2018) 362:339-43.

10. Rothstein M, Bhattacharya D, Simoes-Costa M. The molecular basis of neural crest axial identity. Dev Biol (2018). doi: 10.1016/j.ydbio.2018.07.026. [Epub ahead of print].

11. Gouignard N, Andrieu C, Theveneau E. Neural crest delamination and migration: looking forward to the next 150 years. Genesis (2018) 56:e23107. doi: $10.1002 /$ dvg.23107

12. Pla P, Monsoro-Burq AH. The neural border: induction, specification and maturation of the territory that generates neural crest cells. Dev Biol. (2018). doi: 10.1016/j.ydbio.2018.05.018. [Epub ahead of print].

13. Epperlein HH, Krotoski D, Halfter W, Frey A. Origin and distribution of enteric neurones in Xenopus. Anat Embryol. (1990) 182:53-67. doi: 10.1007/BF00187527

14. Huang X, Saint-Jeannet JP. Induction of the neural crest and the opportunities of life on the edge. Dev Biol. (2004) 275:1-11. doi: 10.1016/j.ydbio.2004.07.033

15. Das BC, Thapa P, Karki R, Das S, Mahapatra S, Liu TC, et al. Retinoic acid signaling pathways in development and diseases. Bioorg Med Chem. (2014) 22:673-83. doi: 10.1016/j.bmc.2013.11.025

16. Glass CK, Rosenfeld MG. The coregulator exchange in transcriptional functions of nuclear receptors. Genes Dev. (2000) 14:121-41. doi: $10.1101 / \operatorname{gad} .14 .2 .121$

17. Dilworth FJ, Fromental-Ramain C, Yamamoto K, Chambon P. ATP-driven chromatin remodeling activity and histone acetyltransferases act sequentially 
during transactivation by RAR/RXR in vitro. Mol Cell (2000) 6:1049-58. doi: 10.1016/S1097-2765(00)00103-9

18. Urvalek AM, Gudas LJ. Retinoic acid and histone deacetylases regulate epigenetic changes in embryonic stem cells. J Biol Chem. (2014) 289:19519-30. doi: 10.1074/jbc.M114.556555

19. Lin RJ, Nagy L, Inoue S, Shao W, Miller WH Jr, Evans RM. Role of the histone deacetylase complex in acute promyelocytic leukaemia. Nature (1998) 391:811-4. doi: 10.1038/35895

20. Delvecchio M, Gaucher J, Aguilar-Gurrieri C, Ortega E, Panne D. Structure of the p300 catalytic core and implications for chromatin targeting and HAT regulation. Nat Struct Mol Biol. (2013) 20: 1040-6. doi: 10.1038/nsmb.2642

21. Lee S, Lee B, Lee JW, Lee SK. Retinoid signaling and neurogenin2 function are coupled for the specification of spinal motor neurons through a chromatin modifier CBP. Neuron (2009) 62:641-54. doi: 10.1016/j.neuron.2009.04.025

22. Léger H, Smet-Nocca C, Attmane-Elakeb A, Morley-Fletcher S, Benecke AG, Eilebrecht S. A TDG/CBP/RARalpha ternary complex mediates the retinoic acid-dependent expression of DNA methylation-sensitive genes. Genom Proteom Bioinform. (2014) 12:8-18. doi: 10.1016/j.gpb.2013.11.001

23. Chen W, Jia W, Wang K, Si X, Zhu S, Duan T, et al. Distinct roles for CBP and p300 on the RA-mediated expression of the meiosis commitment gene Stra8 in mouse embryonic stem cells. PLoS ONE (2013) 8:e66076. doi: 10.1371/journal.pone.0066076

24. Fu M, Sato Y, Lyons-Warren A, Zhang B, Kane MA, Napoli JL, et al. Vitamin A facilitates enteric nervous system precursor migration by reducing Pten accumulation. Development (2010) 137:631-40. doi: 10.1242/dev.040550

25. Niederreither K, Vermot J, Le Roux I, Schuhbaur B, Chambon P, Dollé P. The regional pattern of retinoic acid synthesis by RALDH2 is essential for the development of posterior pharyngeal arches and the enteric nervous system. Development (2003) 130:2525-34. doi: 10.1242/dev.00463

26. Cui J, Michaille JJ, Jiang W, Zile MH. Retinoid receptors and vitamin A deficiency: differential patterns of transcription during early avian development and the rapid induction of RARs by retinoic acid. Dev Biol. (2003) 260:496-511. doi: 10.1016/S0012-1606(03)00257-4

27. Simkin JE, Zhang D, Rollo BN, Newgreen DF. Retinoic acid upregulates ret and induces chain migration and population expansion in vagal neural crest cells to colonise the embryonic gut. PLoS ONE (2013) 8:e64077. doi: 10.1371/journal.pone.0064077

28. Mograbi B, Bocciardi R, Bourget I, Busca R, Rochet N, Farahi-Far D, et al. Glial cell line-derived neurotrophic factor-stimulated phosphatidylinositol 3-kinase and Akt activities exert opposing effects on the ERK pathway: importance for the rescue of neuroectodermic cells. J Biol Chem. (2001) 276:45307-19. doi: 10.1074/jbc.M101220200

29. Asai N, Fukuda T, Wu Z, Enomoto A, Pachnis V, Takahashi M, et al. Targeted mutation of serine 697 in the Ret tyrosine kinase causes migration defect of enteric neural crest cells. Development (2006) 133:450716. doi: $10.1242 / \mathrm{dev} .02616$

30. Chalazonitis A, Rothman TP, Chen J, Gershon MD. Age-dependent differences in the effects of GDNF and NT-3 on the development of neurons and glia from neural crest-derived precursors immunoselected from the fetal rat gut: expression of GFRalpha-1 in vitro and in vivo. Dev Biol. (1998) 204:385-406. doi: 10.1006/dbio.1998.9090

31. Hearn CJ, Murphy M, Newgreen D. GDNF and ET-3 differentially modulate the numbers of avian enteric neural crest cells and enteric neurons in vitro. Dev Biol. (1998) 197:93-105. doi: 10.1006/dbio.1998.8876

32. Heuckeroth RO, Lampe PA, Johnson EM, Milbrandt J. Neurturin and GDNF promote proliferation and survival of enteric neuron and glial progenitors in vitro. Dev Biol. (1998) 200:116-29. doi: 10.1006/dbio.1998.8955

33. Barlow A, de Graaff E, Pachnis V. Enteric nervous system progenitors are coordinately controlled by the G protein-coupled receptor EDNRB and the receptor tyrosine kinase RET. Neuron (2003) 40:905-16. doi: 10.1016/S0896-6273(03)00730-X

34. Li Y, Kido T, Garcia-Barcelo MM, Tam PK, Tabatabai ZL, Lau YF. SRY interference of normal regulation of the RET gene suggests a potential role of the Y-chromosome gene in sexual dimorphism in Hirschsprung disease. Hum Mol Genet. (2015) 24:685-97. doi: 10.1093/hmg/ddu488

35. Shi Y, Li J, Chen C, Gong M, Chen Y, Liu Y, et al. 5-Mehtyltetrahydrofolate rescues alcohol-induced neural crest cell migration abnormalities. Mol Brain (2014) 7:67. doi: 10.1186/s13041-014-0067-9

36. Sato T, Sasai N, Sasai Y. Neural crest determination by co-activation of Pax3 and Zicl genes in Xenopus ectoderm. Development (2005) 132:2355-63. doi: 10.1242/dev. 01823

37. Sasai N, Mizuseki K, Sasai Y. Requirement of FoxD3-class signaling for neural crest determination in Xenopus. Development (2001) 128:2525-36.

38. Aoki Y, Saint-Germain N, Gyda M, Magner-Fink E, Lee YH, Credidio C, et al. Sox10 regulates the development of neural crest-derived melanocytes in Xenopus. Dev Biol. (2003) 259:19-33. doi: 10.1016/S0012-1606(03)00161-1

39. Oschwald R, Richter K, Grunz H. Localization of a nervous system-specific class II beta-tubulin gene in Xenopus laevis embryos by whole-mount in situ hybridization. Int J Dev Biol. (1991) 35:399-405.

40. Yang S, Donner LR. Detection of ganglion cells in the colonic plexuses by immunostaining for neuron-specific marker NeuN: an aid for the diagnosis of Hirschsprung disease. Appl Immunohistochem Mol Morphol. (2002) 10:21820. doi: 10.1097/00129039-200209000-00005

41. Wolf HK, Buslei R, Schmidt-Kastner R, Schmidt-Kastner PK, Pietsch T, Wiestler OD, et al. NeuN: a useful neuronal marker for diagnostic histopathology. J Histochem Cytochem. (1996) 44:1167-71. doi: $10.1177 / 44.10 .8813082$

42. Rollo BN, Zhang D, Stamp LA, Menheniott TR, Stathopoulos L, Denham M, et al. Enteric neural cells from Hirschsprung disease patients form ganglia in autologous aneuronal colon. Cell Mol Gastroenterol Hepatol. (2016) 2:92-109. doi: 10.1016/j.jcmgh.2015.09.007

43. Matsuda H, Hirato J, Kuroiwa M, Nakazato Y. Histopathological and immunohistochemical study of the enteric innervations among various types of aganglionoses including isolated and syndromic Hirschsprung disease. Neuropathology (2006) 26:8-23. doi: 10.1111/j.1440-1789.2006.00649.x

44. Volpe A, Alaggio R, Midrio P, Iaria L, Gamba P. Calretinin, betatubulin immunohistochemistry, and submucosal nerve trunks morphology in Hirschsprung disease: possible applications in clinical practice. J Pediatr Gastroenterol Nutr. (2013) 57:780-7. doi: 10.1097/MPG.0b013e3182a934c7

45. Sharpe CR. Two isoforms of retinoic acid receptor alpha expressed during Xenopus development respond to retinoic acid. Mech Dev. (1992) 39:81-93. doi: 10.1016/0925-4773(92)90028-I

46. Bondurand N, Sham MH. The role of SOX10 during enteric nervous system development. Dev Biol. (2013) 382:330-43. doi: 10.1016/j.ydbio.2013.04.024

47. Hou N, Ren L, Gong M, Bi Y, Gu Y, Dong Z, et al. Vitamin A deficiency impairs spatial learning and memory: the mechanism of abnormal CBP-dependent histone acetylation regulated by retinoic acid receptor alpha. Mol Neurobiol. (2015) 51:633-47. doi: 10.1007/s12035-014-8741-6

48. Dietze EC, Caldwell LE, Marcom K, Collins SJ, Yee L, Swisshelm K, et al. Retinoids and retinoic acid receptors regulate growth arrest and apoptosis in human mammary epithelial cells and modulate expression of CBP/p300. Microsc Res Tech. (2002) 59:23-40. doi: 10.1002/jemt.10174

49. Wang WD, Melville DB, Montero-Balaguer M, Hatzopoulos AK, Knapik EW. Tfap2a and Foxd3 regulate early steps in the development of the neural crest progenitor population. Dev Biol. (2011) 360:173-85. doi: 10.1016/j.ydbio.2011.09.019

50. Monsoro-Burq AH, Fletcher RB, Harland RM. Neural crest induction by paraxial mesoderm in Xenopus embryos requires FGF signals. Development (2003) 130:3111-24. doi: 10.1242/dev.00531

51. Gammill LS, Bronner-Fraser M. Genomic analysis of neural crest induction. Development (2002) 129:5731-41. doi: 10.1242/dev.00175

52. Lake JI, Heuckeroth RO. Enteric nervous system development: migration, differentiation, and disease. Am J Physiol Gastrointest Liver Physiol. (2013) 305:G1-24. doi: 10.1152/ajpgi.00452.2012

53. Koide T, Downes M, Chandraratna RA, Blumberg B, Umesono K. Active repression of RAR signaling is required for head formation. Genes Dev. (2001) 15:2111-21. doi: 10.1101/gad.908801

Conflict of Interest Statement: The authors declare that the research was conducted in the absence of any commercial or financial relationships that could be construed as a potential conflict of interest.

Copyright $\odot 2018$ Li, Hu, Hou, Wang, Wang, Yang, Gu, He, Shi, Chen, Song and Li. This is an open-access article distributed under the terms of the Creative Commons Attribution License (CC BY). The use, distribution or reproduction in other forums is permitted, provided the original author(s) and the copyright owner(s) are credited and that the original publication in this journal is cited, in accordance with accepted academic practice. No use, distribution or reproduction is permitted which does not comply with these terms. 